extrinsic coagulation defects, intra-abdominal infection \& congenital abnormalities. These analyses were normal with diagnosis PVT of unknown cause; over $50 \%$ of cases have no known aetiology ${ }^{[2]}$. DH had complications including ascites, splenomegaly \& an oesophageal varix. He will need regular OGD follow up to check if change to varix or development of new varices, $79 \%$ of children with PVT have one upper gastrointestinal bleed in their lives ${ }^{[3]}$.

Conclusion Ascites is an unusual presentation with specific investigations. PVT is a rare cause of ascites with complications that require investigation.

[1] Karnasakul W, Thammasin I et al. Ascites in Children: A Single-Centre Experience of 27 years. Journal Paediatric Gastroenterology, Hepatology \& Nutrition, 64 (1),, 83-88. 2017

[2] Weiss B, Shteyer E, Vivante A, Berkowitz D, Reif S, Weizman Z, Bujanover Y, Shapiro R. Etiology and long-term outcome of extrahepatic portal vein obstruction in children. World J Gastroenterol. $2010 ; 16: 4968-72$.

[3] Ferri, Menezes P., et al. Portal vein thrombosis in children and adolescents: 20 years experience of a pediatric hepatology reference center. Arquivos de Gastroenterologia, 49(1), 69-76. 2012

\section{P334 CONGENITAL CYTOMEGALOVIRUS INFECTION}

\footnotetext{
${ }^{1,2}$ Alina Grama, ${ }^{2}$ Claudia Sirbe, ${ }^{2}$ Madalina Grigore, ${ }^{2}$ Adriana Bungardi, ${ }^{1,2}$ Tudor L Pop*. ${ }^{1}$ University of Medicine and Pharmacy 'Iuliu Hatieganu', Cluj-Napoca, Romania; ${ }^{2} 2 n d$ Paediatric Clinic, Cluj-Napoca, Romania
}

\subsection{6/archdischild-2019-epa.683}

Introduction Congenital cytomegalovirus (CMV) infection is the most common congenital infection, with an incidence of $0.5 \%$ among neonates. Mother to child CMV transmission could be transplacental (mostly), at delivery or after birth, through exposure to breast milk. The clinical picture may range from asymptomatic disease to severe form of illness, sometimes occurred immediately after birth. These symptomatic infants have a high risk for neurologic sequelae, including sensorineural hearing loss, mental retardation, microcephaly, development delay, seizure disorders, and cerebral palsy. Congenital CMV is the most common cause of acquired hearing loss in childhood.

Clinical cases We report the clinical and laboratory parameters of three patients with severe congenital CMV infection. In all the maternal infection was asymptomatic and TORCH serology before/during pregnancy was not performed.

The first patient was a preterm boy (born at 28 weeks of gestation with the weight of $1,190 \mathrm{~g}$ ) with cholestatic hepatitis and complex perinatal pathology, necrotizing enterocolitis, recurrent episodes of apnoea and bradycardia, severe anaemia and thrombocytopenia in the context of CMV infection. The second patient was a girl, born at 37 weeks of gestation with intrauterine growth retardation (weight 2,420 g), who developed immediately after birth CMV pneumoniae, cholestatic hepatitis and severe cytopenia. Also, the audiogram was suggestive for hearing impairment. The third patient was a preterm girl, born at 35 weeks of gestation, weight 2,600 g, with cerebral vasculopathy and splenomegaly after intrauterine CMV infection.

In all these patients, the infection was confirmed by positive serological IgM-CMV antibodies and by quantitative CMV
PCR assay in plasma and all of them received antiviral therapy with ganciclovir.

Conclusions Congenital CMV is an important cause of morbidity in children. Early detection of CMV infection by CMV PCR or culture from saliva or urine and a prompt treatment can prevent life threatening complications like acute liver failure, pneumonitis or sepsis-like syndrome or can improve hearing and neurological development. All children with congenital CMV should be periodically monitored to prevent hearing loss or other neurocognitive sequelae.

\section{P335 'LIPID TRIAD ‘ IN PATIENTS WITH CHRONIC PANCREATITIS IN COMBINATION WITH ARTERIAL HYPERTENSION}

Olesya Horlenko*, Emilia Arkhiy, Lyubomyra Prylypko, Oxana Moscal, Maria Derbak , Bogdan Halay. Uzhhorod National Universiry, Uzhhorod, Ukraine

\subsection{6/archdischild-2019-epa.684}

Introduction Modern scientific research in the field of medical knowledge has repeatedly confirmed that the issue of 'comorbidity' is becoming widespread. More and more rare occurrences of the mononozological course of various diseases and the further, the more often - variants of a comorbid or multi-morbid.

Materials In order to achieve this goal, we carried out a general clinical, laboratory and instrumental examination of 102 patients with comorbid pathology of CP and GC that were in the hospital treatment of Khust district hospital during 20172018. The average age of the examined patient was $51 \pm 10$ years.

Results Concerning the features of lipid metabolism, the results obtained by us confirm the presence of 'lipid triad' in patients with Chronic Pancreatitis (CP) in combination with Arterial Hypertension a generalized persistence of inflammatory changes at the level of vascular endothelium. It is difficult to say with certainty that the primary focus in the examined group of patients, or dislipoproteinemia against the background of an existing $\mathrm{CP}$ with $\mathrm{AH}$, but it is possible to clearly state the imbalance in the system of lipid homeostasis, which ultimately changes the activity of all organs and systems. The results of the study, which reproduce the changes in Apolipoprotein levels, were as follows: in the main group, the concentration of Apo A1 decreased to $0.85 \pm 0.11 \mathrm{~g} / \mathrm{l}$ against $1.08 \pm 0.12 \mathrm{~g} / \mathrm{l}$ in the control group $(\mathrm{p}<0,05)$, and the excessive level of proatherogenic $A p o B$ in the group of patients with $\mathrm{CP}$ and $\mathrm{AH}-1.46 \pm 0.23 \mathrm{~g} / \mathrm{l}$, and in practically healthy individuals $-0.99 \pm 0.24 \mathrm{~g} / \mathrm{l}(\mathrm{p}<0,05)$

In addition to the absolute values of lipidogram rates, in order to assess the presence or potential risk of developing atherosclerotic vascular damage, the ratio of Apo B/Apo A1 and the atherogenicity index that were higher in the group of patients with CP and GC: Apo B/Apo A1 - 1, $77 \pm 0.46$ versus $0.92 \pm 0.14$ (in the control group $(\mathrm{p}<0,05)$ and IA - in the I group $-5,42 \pm 2,25$ versus $2,4 \pm 1,02(\mathrm{p}<0,05)$. The latter indicators are necessary to determine the further treatment of patients and control of the prescribed therapy effectiveness.

Conclusion The results of the study carried out during the hospitalization period in the main group indicate an increase in concentrations of proatherogenic fractions of lipids and apolipoproteins (LDL, LPDH, Apo B) and reduction of antiatherogenic (HDL, Apo A1). The atherogenicity index and the 